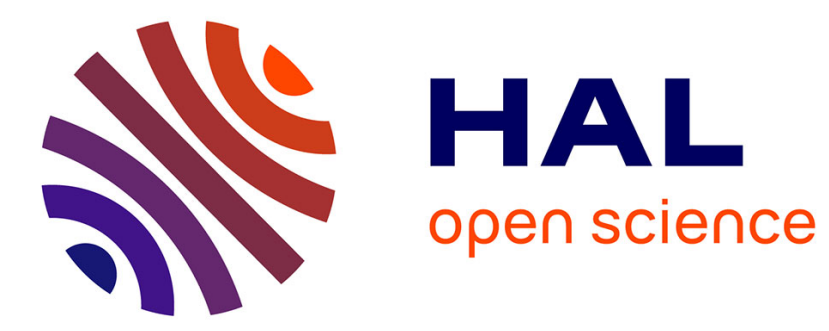

\title{
Ultrasonic attenuation in the amorphous metals Nip and PdSi at low temperatures
}

\author{
P. Doussineau, A. Levelut, G. Bellessa, O. Bethoux
}

\section{To cite this version:}

P. Doussineau, A. Levelut, G. Bellessa, O. Bethoux. Ultrasonic attenuation in the amorphous metals Nip and PdSi at low temperatures. Journal de Physique Lettres, 1977, 38 (24), pp.483-487. 10.1051/jphyslet:019770038024048300 . jpa-00231426

\section{HAL Id: jpa-00231426 https://hal.science/jpa-00231426}

Submitted on 1 Jan 1977

HAL is a multi-disciplinary open access archive for the deposit and dissemination of scientific research documents, whether they are published or not. The documents may come from teaching and research institutions in France or abroad, or from public or private research centers.
L'archive ouverte pluridisciplinaire HAL, est destinée au dépôt et à la diffusion de documents scientifiques de niveau recherche, publiés ou non, émanant des établissements d'enseignement et de recherche français ou étrangers, des laboratoires publics ou privés. 


\title{
ULTRASONIC ATTENUATION IN THE AMORPHOUS METALS NiP AND PdSi AT LOW TEMPERATURES
}

\author{
P. DOUSSINEAU, A. LEVELUT
}

Laboratoire d'Ultrasons $\left({ }^{*}\right)$, Université Pierre-et-Marie-Curie, Tour 13, 4 place Jussieu, 75230 Paris Cedex 05, France

\section{G. BELLESSA}

Laboratoire de Physique des Solides (*), Université Paris-Sud, Bâtiment 510, 91405 Orsay, France

and

\section{O. BETHOUX}

Centre de Recherches sur les Très Basses Températures, C.N.R.S., 166 X, Centre de tri, 38042 Grenoble Cedex, France

(Reçu le 17 octobre 1977, accepté le 18 novembre 1977)

\begin{abstract}
Résumé. - L'atténuation d'ondes ultrasonores longitudinales a été mesurée dans les alliages métalliques amorphes $\mathrm{Ni}_{0,78} \mathrm{P}_{0,22}$ et $\mathrm{Pd}_{0,775} \mathrm{Si}_{0,165} \mathrm{Cu}_{0,06}$ dans la gamme de température 0,6 à $7 \mathrm{~K}$ pour différentes fréquences comprises entre 0,6 et $2 \mathrm{GHz}$. Les résultats dans les deux matériaux sont bien décrits par une loi de la forme $\alpha=\alpha_{0}(\omega)+A \omega T+B \omega^{2} \log \frac{T}{T_{0}}$. Ce comportement est comparé aux résultats obtenus dans les isolants et aux prédictions des théories de l'atténuation ultrasonore par les systèmes à deux niveaux et les électrons. On montre que l'atténuation ultrasonore dans les métaux amorphes est différente de celle observée dans les cristaux et dans les verres isolants.

Abstract. - Attenuation of longitudinal ultrasonic waves has been measured in the amorphous metallic alloys $\mathrm{Ni}_{0.78} \mathrm{P}_{0.22}$ and $\mathrm{Pd}_{0.775} \mathrm{Si}_{0.165} \mathrm{Cu}_{0.06}$ in the temperature range $0.6-7 \mathrm{~K}$ for various frequencies between 0.6 and $2 \mathrm{GHz}$. In the two materials the results are well described by a law of the form : $\alpha=\alpha_{0}(\omega)+A \omega T+B \omega^{2} \log \frac{T}{T_{0}}$. This behaviour is compared with the results obtained in glasses and with the predictions of the theories of the ultrasonic attenuation by the two-level systems and the electrons. It is shown that the ultrasonic attenuation in amorphous metals is different from that observed in crystals and amorphous insulators.
\end{abstract}

1. Introduction. - Over the past few years considerable information has been obtained on the low temperature properties of amorphous insulators $[1,2]$. They present an anomalous behaviour for specific heat, thermal conductivity and sound propagation; it is explained by the existence of localized excitations in form of two-level system (TLS) with a nearly constant density of states [3]. The sound propagation measurements are particularly appropriate for the study of amorphous materials because of the high coupling between the acoustical wave and the TLS. The main features observed are the saturation of the sound absorption and the logarithmic temperature dependence of the sound velocity [2].

$\left(^{*}\right)$ Associated with the Centre National de la Recherche Scientifique.
The anomalies observed in amorphous insulators present an universal character, not only qualitatively but also quantitatively. It seems that the thermodynamic and elastic properties of glasses pertain rather to the glassy state than to the chemical composition of the material.

The problem now is to determine whether the same type of TLS exists in other amorphous materials. Recently TLS have been invoked in the interpretation of resistivity [4] and thermal conductivity [5] measurements in some amorphous metallic alloys. An excess specific heat in the glassy state has also been measured but its origin is not clearly attributed [6]. In fact, in the later two cases there is some problem in the evaluation of the electronic contribution. The observation of a logarithmic temperature dependence of the sound velocity in amorphous NiP [7], CoP [8], PdSi [9] 
and $\mathrm{Se}[10]$, has unambiguously proved the existence of TLS in these materials. One of the main interests for the study of amorphous metals lies in their more compact structure. The difference in density between the amorphous and crystalline states is $20 \%$ for silica and about $2 \%$ for metals. In the first case (silica), one may imagine that atoms can more easily move and oscillate between two positions. Therefore, it is hoped that the predictions of the tunnelling model must have differents orders of magnitude in glasses and in amorphous metals. The sound velocity measurements are a first answer to this conjecture.

2. Samples and experimental techniques. - The $\mathrm{NiP}$ samples, with a composition $\mathrm{Ni}_{0.78} \mathrm{P}_{0.22}$, are electrodeposited from the bath. They are in the shape of platelets about $1 \mathrm{~mm}$ thick. With this composition the alloy NiP is non-magnetic.

The samples of palladium-silicon alloy are obtained from the liquid phase by a rapid quenching. Their composition is $\mathrm{Pd}_{0.775} \mathrm{Si}_{0.165} \mathrm{Cu}_{0.06}$. They have the shape of a rod of diameter $2 \mathrm{~mm}$.

Ultrasonic waves of frequency in the gigahertz range were generated by a thin film of $\mathrm{ZnO}$ or $\mathrm{LiNbO}_{3}$ deposited on a corundum rod. The sample was bounded to the corundum rod with Dow Corning grease. The experiments were performed by using a standard pulse-echo technique.

The change in the sound attenuation was measured by recording the variation of the amplitude of a given echo, or sometimes two successive echoes, as the temperature was varied. The error was $\pm 0.1 \mathrm{~dB} \mathrm{~cm}^{-1}$. The acoustic flux in the sample was estimated to be about $10^{-4} \mathrm{~W} \mathrm{~cm}^{-2}$. The temperature was measured by a carbon resistor.

3. Experimental results. - The attenuation of longitudinal waves has been measured in amorphous $\mathrm{NiP}$ and $\mathrm{PdSi}$ in the temperature range $0.6<T<7 \mathrm{~K}$ for various frequencies between 600 and $2000 \mathrm{MHz}$. For one experiment on $\mathrm{PdSi}$ the temperature range was extended up to $30 \mathrm{~K}$.

The measured ultrasonic attenuation as a function of the temperature is given in figure 1 for $\mathrm{NiP}$ at three ultrasonic frequencies and in figure 2 for $\mathrm{PdSi}$ for two frequencies. For comparison we have plotted in figure 3 the results for the two amorphous metals and for a glass : Tetrasil A (a variety of silica with 1000 ppm OH).

The main features of these results are the following :

- No power dependence of the attenuation has been observed over a range of acoustic flux from $10^{-7}$ to $10^{-4} \mathrm{~W} \mathrm{~cm}^{-2}$.

- In the temperature range $2<T<7 \mathrm{~K}$ the main contribution to the attenuation depends linearly on the temperature $T$; moreover the slope of the curves $\alpha(T)$ depends linearly on the frequency $\omega / 2 \pi$.

- At the lowest temperatures (below $2 \mathrm{~K}$ ) and the highest frequencies (above $1000 \mathrm{MHz}$ ) another

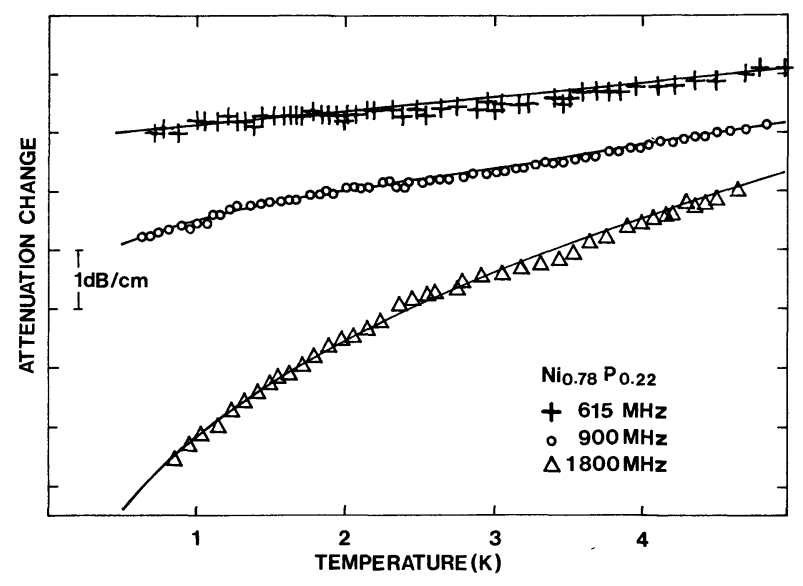

FIG. 1. - Variation of the attenuation of an ultrasonic wave propagating in amorphous $\mathrm{NiP}$ as a function of the temperature for three different frequencies. The curves are arbitrarily shifted one from another. The continuous lines are calculated curves with an expression of the form : $\alpha=\alpha_{0}(\omega)+A \omega T+B \omega^{2} \log \frac{T}{T_{0}}$ (see text). The value of $\alpha_{0}(\omega)$ is chosen so that $\alpha_{\text {cal }}$ and $\alpha_{\text {exp }}$ are equal at $T=1 \mathrm{~K}$.

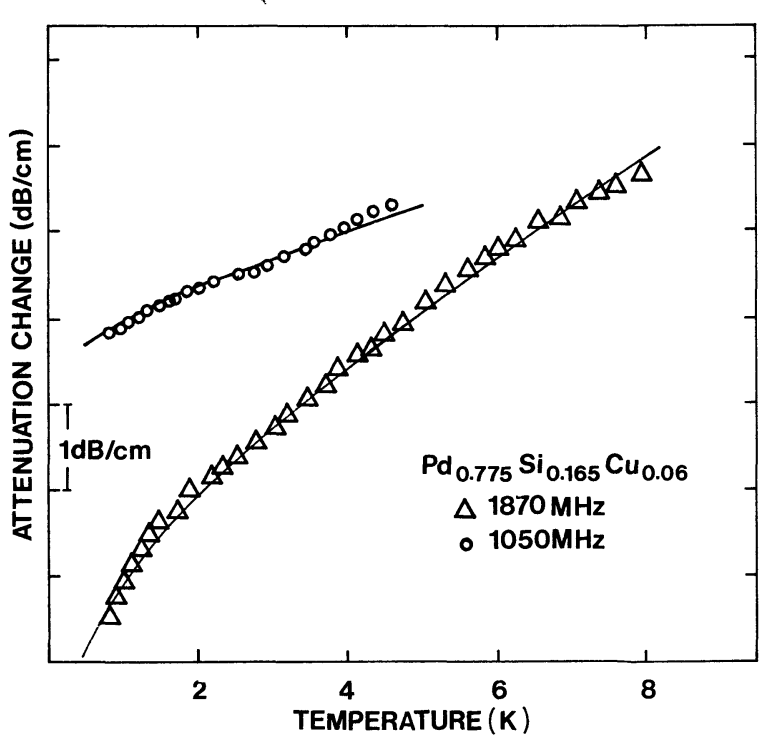

FIG. 2. - Variation of the attenuation of an ultrasonic wave propagating in amorphous $\mathrm{PdSi}$ as a function of the temperature for two different frequencies. The curves are arbitrarily shifted one from another. The continuous lines are calculated curves with an expression of the form : $\alpha=\alpha_{0}(\omega)+A \omega T+B \omega^{2} \log \frac{T}{T_{0}}$ (see text). The value of $\alpha_{0}(\omega)$ is chosen so that $\alpha_{\text {cal }}$ and $\alpha_{\text {exp }}$ are equal at $T=1 \mathrm{~K}$.

regime becomes important (Figs. 1 and 2). Its contribution can be described by an $\omega^{2} \log T$ law.

- The behaviour of the two materials NiP and PdSi is remarkably similar. Not only are the temperature and frequency dependence of the sound attenuation identical, but also the magnitude is nearly the same.

- Figure 3 clearly shows the difference of the sound attenuation between amorphous metals and 


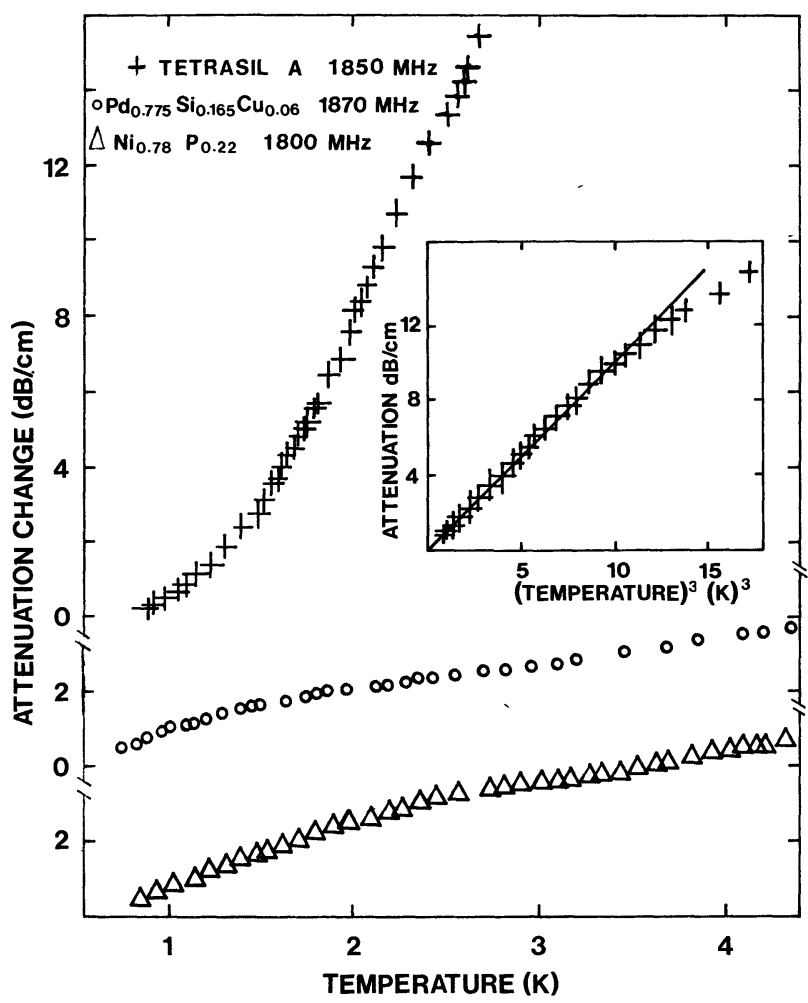

FIG. 3. - Variation of the attenuation of an ultrasonic wave propagating in three different amorphous materials (two metals NiP and PdSi and one insulator silica Tetrasil A) as a function of the temperature at neighbouring frequencies. In the three cases the the acoustic flux in the sample is about $10^{-4} \mathrm{~W} \mathrm{~cm}^{-2}$. The curves are arbitrarily shifted one from another. The zero of the attenuation is arbitrarily fixed. The insert shows the attenuation in Tetrasil A plotted as a function of the cube of the temperature.

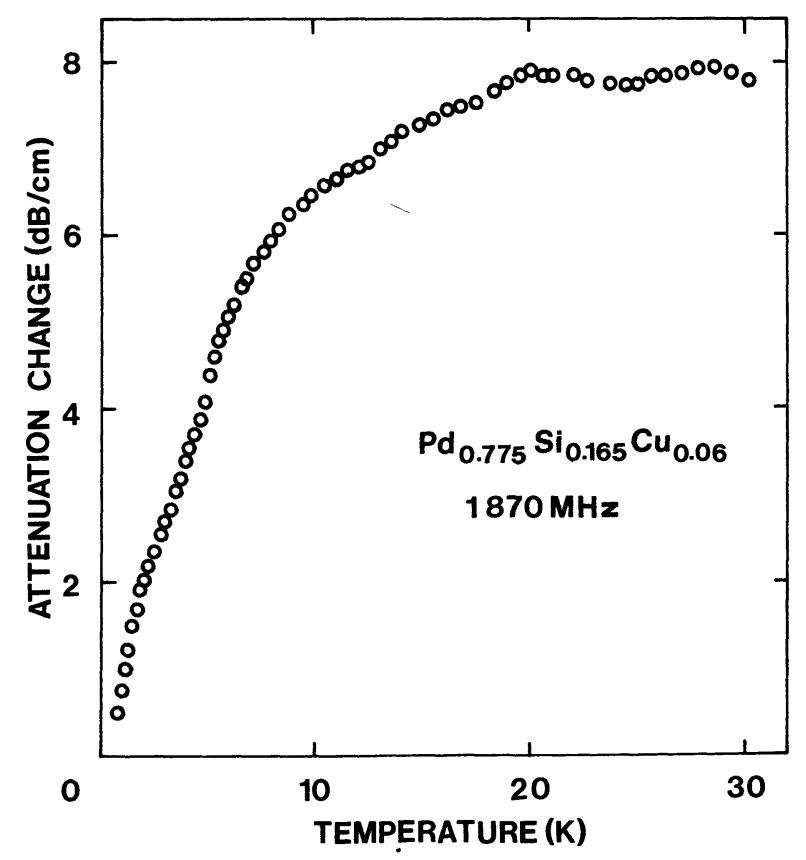

FIG. 4. - Variation of the attenuation of an ultrasonic wave propagating in a PdSi sample as a function of temperature between 1 and $30 \mathrm{~K}$. The zero of the attenuation is arbitrarily fixed. insulators. In amorphous insulators, at high acoustic intensity the attenuation (due to non-resonant processes) is proportional to $\omega^{0} T^{3}$. This behaviour is clearly seen in our experiment on Tetrasil A at $1850 \mathrm{MHz}$ (see insert in Fig. 3), and the magnitude is in good agreement with earlier experiments in silica or borosilicate at lower [12] and higher [13] frequencies.

- In one experimental run the attenuation of $1870 \mathrm{MHz}$ sound wave was measured in $\mathrm{PdSi}$ up to $30 \mathrm{~K}$ (Fig. 4). A linear temperature dependence is observed up to $7 \mathrm{~K}$. For $T>7 \mathrm{~K}$, the attenuation increases more slowly, roughly linearly but with a weaker slope than is measured at lower temperatures. It reaches a nearly constant value at $20 \mathrm{~K}$ up to $30 \mathrm{~K}$. The behaviour in this temperature range has not been as carefully studied as at lower temperatures. But previous experiments [14] at the same sound frequencies and in the same temperature range in amorphous $\mathrm{Pd}_{0.775} \mathrm{Si}_{0.165} \mathrm{Ag}_{0.06}$ have given similar results. This gives a good check for the validity of our results.

We have fitted our results with an empirical law of the form :

$$
\alpha=\alpha_{0}(\omega)+A \omega T+B \omega^{2} \log \frac{T}{T_{0}}
$$

which is valid (at least) in the range $0.6<T<7 \mathrm{~K}$, $500 \mathrm{MHz}<\frac{\omega}{2 \pi}<2000 \mathrm{MHz} . T_{0}$ is an arbitrary temperature reference chosen to be $1 \mathrm{~K} . \alpha_{0}(\omega)$ is the temperature independent part of the attenuation probably due to electrons, impurities, defects... Its value cannot be determined in our experiments because we have measured an attenuation change, not an absolute value.

The best fit is obtained with

$$
\left.\begin{array}{l}
A=1.1 \times 10^{-11} \mathrm{~cm}^{-1} \mathrm{~K}^{-1}(\mathrm{rad} / \mathrm{s})^{-1} \\
B=6.0 \times 10^{-21} \mathrm{~cm}^{-1}(\mathrm{rad} / \mathrm{s})^{-2}
\end{array}\right\} \text { for } \mathrm{NiP}
$$

and

$$
\left.\begin{array}{l}
A=0.9 \times 10^{-11} \mathrm{~cm}^{-1} \mathrm{~K}^{-1}(\mathrm{rad} / \mathrm{s})^{-1} \\
B=3.0 \times 10^{-21} \mathrm{~cm}^{-1}(\mathrm{rad} / \mathrm{s})^{-2}
\end{array}\right\} \text { for PdSi. }
$$

The accuracy is about $10 \%$.

The curves calculated with these values are the continuous lines in figures 1 and 2 . For each material, the same values of the parameters $A$ and $B$ have been used for all frequencies.

4. Discussion. - These experimental results may be compared with those obtained in amorphous insulators and with the predictions of the existing theories of the sound attenuation.

However, we first mention sound propagation measurements in amorphous PdSi recently reported for frequencies around $1000 \mathrm{MHz}$ and for temperatures between $0.015 \mathrm{~K}$ and a few $\mathrm{K}$ [15]. In the tempe- 
rature range around $1 \mathrm{~K}$ (in common with our results) the experimental results are fitted with a law of the form $B \omega^{2} \log \frac{T}{T_{0}}$ with $B=7.2 \times 10^{-21} \mathrm{~cm}^{-1}(\mathrm{rad} /$ $s)^{-2}$. If we compare our results to the same law (without the term $A \omega T$ ) we obtain a value of the parameter $B=6 \times 10^{-21} \mathrm{~cm}^{-1}(\mathrm{rad} / \mathrm{s})^{-2}$. Therefore the two results are consistent.

The questions which must be answered in the discussion of the sound attenuation in amorphous metals are the following :

- Is the coupling between the sound wave and the TLS responsible for the attenuation as well as for the logarithmic temperature dependence of the sound velocity ?

- What is the role of the electrons?

4.1 Sound attenuation By THE TLS. - The coupling between TLS and sound wave leads to two well-known different contributions to the attenuation of the sound wave. The first process is a resonant one with absorption or emission of one phonon [2]. The corresponding attenuation is given by :

$$
l_{\mathrm{res}}^{-1}=\frac{\pi n_{0} G_{x}^{2}}{4 \rho v^{3}\left(1+\frac{\Phi}{\Phi_{\mathrm{c}}}\right)^{1 / 2}} \omega \tanh \left(\frac{\hbar \omega}{2 k T}\right)
$$

where $n_{0}$ is the (constant) density of states of the TLS, $G_{x}$ the off-diagonal coupling coefficient between the TLS and phonons, $\omega / 2 \pi$ the frequency of the sound wave of flux $\Phi$ propagating in the amorphous sample of density $\rho$ with a velocity $v$; $\Phi_{c}$ is the critical flux above which the saturation effect becomes important.

The observation of a logarithmic temperature dependence of the sound velocity in $\mathrm{NiP}$ [7] and $\mathrm{PdSi}$ [9] proves the existence of TLS in these materials and provides us with the values of $\frac{n_{0} G_{x}^{2}}{4 \rho v^{2}}$. Therefore we can calculate the resonant part of the attenuation : it is found to be weak (at $1 \mathrm{~K}$ and $2000 \mathrm{MHz}, 6 \times 10^{-2}$ $\mathrm{dB} \mathrm{cm} \mathrm{cm}^{-1}$ for NiP and $5 \times 10^{-2} \mathrm{~dB} \mathrm{~cm}^{-1}$ for PdSi). These low values explain why we observed neither power dependence nor resonant attenuation both in $\mathrm{NiP}$ and $\mathrm{PdSi}$. Recent experiments on PdSi at very low temperatures [15] show an increase of the attenuation explained by resonant process with TLS in this material as in glasses : at $15 \mathrm{mK}$ that process is enhanced by a factor 40 when it is compared to our lowest temperature $(0.6 \mathrm{~K})$. Therefore the two results are not contradictory. Moreover no saturation effect have been detected. One consequence of these results is the high value of the critical acoustic flux $\Phi_{\mathrm{c}}$ and therefore the low value of the product $T_{1} T_{2}$. ( $T_{1}$ is the longitudinal relaxation time of the TLS population and $T_{2}$ the irreversible transverse relaxation time). A short $T_{1}$ is given by the relaxation of TLS by electrons (see below).
The second process leads to a non-resonant attenuation due to the relaxation of the TLS population [2]. The attenuation caused by this process is given by :

$$
\begin{aligned}
& l_{\mathrm{rel}}^{-1}=\frac{n_{0} G_{z}^{2}}{4 \rho v^{3}} \times \\
& \quad \times \int_{0}^{\infty} \frac{\hbar}{k T} \operatorname{sech}^{2}\left(\frac{\hbar \omega_{0}}{2 k T}\right) \frac{\omega^{2} T_{1}\left(\omega_{0}\right)}{1+\omega^{2} T_{1}^{2}\left(\omega_{0}\right)} \mathrm{d} \omega_{0}
\end{aligned}
$$

where $G_{z}$ is the diagonal coupling coefficient between the TLS and sound wave, $\omega_{0}$ the level splitting of the TLS, $T_{1}\left(\omega_{0}\right)$ the relaxation time of the TLS population which, if it is governed by the interaction of TLS with thermal phonons, is given by [2] :

$$
T_{1}^{-1}\left(\omega_{0}\right)=\left(\frac{G_{x, 1}^{2}}{v_{1}^{5}}+\frac{2 G_{x, \mathrm{t}}^{2}}{v_{\mathrm{t}}^{5}}\right) \frac{\omega_{0}^{3}}{8 \pi \rho \hbar} \operatorname{cotanh}\left(\frac{\hbar \omega_{0}}{2 k T}\right)
$$

1 and $t$ refer to the longitudinal and transverse polarizations. In the low temperature regime $\left(\omega T_{1} \gg 1\right)$ this attenuation is frequency independent and depends on the temperature as $T^{3}$.

This contribution is clearly seen in the amorphous insulator Tetrasil A (Fig. 3) in the temperature range $(0.8<T<2.8 \mathrm{~K})$. It is obvious that our results in the amorphous metals NiP and PdSi cannot be explained by such a process.

4.2 SOUND ATTENUATION BY THE ELECTRONS. The electrical conductivity of these materials is relatively large $\left(10^{4} \Omega^{-1} \mathrm{~cm}^{-1}\right.$ at $4.2 \mathrm{~K}$ for $\mathrm{NiP}$ [4] and about $0.7 \times 10^{4} \Omega^{-1} \mathrm{~cm}^{-1}$ at room temperature for $\mathrm{PdSi}$ ) and is nearly temperature independent. Therefore an electronic contribution to the sound attenuation is likely to be important. This contribution has been calculated [16], and varies as $\omega T^{0}$ (when $q l \gg 1$ where $q$ is the phonon wave vector and $l$ the mean free path of the electrons) and as $\omega^{2} \tau$ (when $q l \ll 1$ ) where $\tau$ is the relaxation time of the electrons. In alloys like $\mathrm{PdSi}$ or $\mathrm{NiP}, \tau$ is expected to be temperature independent. Consequently the electrons are not directly the cause of the observed sound attenuation. Their contribution which in any case is weak (about $10^{-2} \mathrm{~dB} / \mathrm{cm}$ ) must be incorporated in the term $\alpha_{0}(\omega)$.

\subsection{OTHER MECHANISM OF SOUND ATTENUATION. -} The previous discussion demonstrates the failure of the usual mechanisms of sound absorption in glasses and crystalline metals to explain our experimental results in amorphous metals. Therefore it is necessary to search for other processes. One possibility may come through the relaxation of the TLS by the electrons. We have calculated, the corresponding relaxation time $T_{1}$ :

$$
T_{1}^{-1}\left(\omega_{0}\right)=\frac{\pi k T}{\hbar} V^{2}\left(\frac{3 N_{\mathrm{a}}}{2 E_{\mathrm{F}}}\right)^{2}
$$


where $V$ is the coupling constant between the TLS and the electrons, $N_{\mathrm{a}}$ the number of conduction electrons per atom and $E_{\mathrm{F}}$ the energy at the Fermi level. This expression is valid if the conditions

$$
\hbar \omega_{0} \ll k T \ll E_{\mathrm{F}}
$$

are fulfilled and $T_{1}\left(\omega_{0}\right)$ is then actually independent of $\omega_{0}$.

In order to obtain a numerical evaluation of $T_{1}$ we have to estimate the value of $V$ which is analogous to the deformation potential $\Delta$ occuring in the electron phonon interaction. We assume $V \sim(l / a) \Delta \sim 10^{-3} \Delta$ where $a$ is an interatomic distance and $l$ the displacement of TLS in the double potential well. Therefore with $\Delta \sim 10 \mathrm{eV}$, we have $V \sim 10^{-2} \mathrm{eV}$. With $N_{\mathrm{a}}=1$ and $E_{\mathrm{F}} \simeq 3 \mathrm{eV}$, we obtain

$$
T_{1}^{-1} \simeq 10^{7} \mathrm{~s}^{-1} \quad \text { at } \quad T=1 \mathrm{~K} .
$$

This is the same order of magnitude than the $T_{1}^{-1}$ of the relaxation of the TLS by thermal phonons at $10 \mathrm{GHz}$ and $T=1 \mathrm{~K}$. The two processes of relaxation are competitive.

The corresponding non-resonant attenuation is calculated as above with the new expression for $T_{1}\left(\omega_{0}\right)$. In the regime $\omega T_{1} \gg 1$ it is proportional to $\omega^{0} T$, and to $\omega^{2} T^{-1}$ when $\omega T_{1} \ll 1$. Therefore this mechanism cannot explain the experimental results in anyone of the two observed regimes $\left(\omega^{2} \log T\right.$ and $\omega T)$.

5. Conclusion. - Recent data have demonstrated that the behaviour of the sound velocity at low temperatures is completely different in a crystalline metal and in a glassy metal. Because the experimental results fit the characteristic law $\Delta v / v \sim \log T$, it has been concluded that TLS exist in amorphous metals.

The results on sound attenuation reported in the present paper also show that crystalline and amorphous metals are quite different from that point of view. But, in contrast to the velocity data, none of the typical attenuation processes of glasses have been recognized. Moreover the classical behaviour of crystalline metals has not been observed.

It may be concluded that glassy metals do attenuate acoustic waves differently from crystalline metals do. TLS probably have a role in the attenuation through a process which has yet to be found. Perhaps a process with one phonon, one electron and one TLS should be invoked. More theoretical calculations are necessary.

Acknowledgments. - We thank B. Golding for helpful discussions and M. Cagnon an J. F. Sadoc for providing us with the NiP samples.

\section{References}

[1] PoHL, R. O., in Phonon Scattering in Solids. L. J. Challis, V. W. Rampton and A. F. G. Wyatt, Eds. (Plenum, New York) 1976, page 107.

[2] Hunklinger, S. and ARnold, W., in Physical Acoustics edited by R. N. Thurston and W. P. Mason (Academic Press, New York) 12 (1976) 155.

[3] Phillips, W. A., J. Low Temp. Phys. 7 (1972) 351

Anderson, P. W., Halperin, B. I. and Varma, C. M., Philos. Mag. 25(1972) 1.

[4] Cochrane, R. W., Harris, R., Ström-Olson, J. O. and Zuckermann, M. J., Phys. Rev. Lett. 35 (1975) 676.

[5] Matey, J. R. and Anderson, A. C., J. Non Cryst. Solids 23 (1977) 129 and to be published.

[6] Golding, B., Bagley, B. G. and Hsu, F. S. L., Phys. Rev. Lett. 29 (1972) 68;

Chen, H. S. and Haemmerle, W. H., J. Non Cryst. Solids 11 (1972) 161.

[7] Bellessa, G., Doussineau, P. and Levelut, A., J. Physique Lett. 38 (1977) L-65.
[8] Bellessa, G., J. Phys. C 10 (1977) L-285.

[9] Bellessa, G. and Bethoux, O., Phys. Lett. 62A (1977) 125. [10] Bellessa, G., Lemercier, C. and Caldemaison, D., Phys. Lett. 62A (1977) 127.

[11] Preliminary work on NiP has been presented at the International Conference on Lattice Dynamics, Paris 5-9 sept. 1977 : Doussineau, P., Levelut, A. and Bellessa, G., to be published.

[12] Jäckle, J., Piché, L., Arnold, W. and Hunklinger, S. J. Non Cryst. Solids. 20 (1976) 365.

[13] Bellessa, G., Cagnon, M., Sadoc, J. F., Doussineau, P. and Levelut, A., J. Physique Lett. 37 (1976) L-291.

[14] Dutort, M. and Chen, H. S., Appl. Phys. Lett. 23 (1973) 357. Dutort, M., Phys. Lett. 50A (1974) 221.

[15] Golding, B., Graebner, J. E. and Haemmerle, W. H., presented at the International Conference on Lattice Dynamics, Paris, 5-9 sept. 1977 : to be published.

[16] PIPPARD, A. B., The Dynamics of Conduction Electrons (Gordon and Breach, New York), 1965. 